\title{
Medindo consumo de álcool: análise fatorial confirmatória do Alcohol Use Disorder Identification Test (AUDIT)
}

\author{
Walberto Silva dos Santos - Universidade Federal do Ceará, Fortaleza, Brasil \\ Darlene Pinho Fernandes - Universidade Federal do Ceará, Fortaleza, Brasil \\ Alex Sandro de Moura Grangeiro - Universidade Federal do Ceará, Fortaleza, Brasil \\ Guilherme Sobreira Lopes - Universidade Federal do Ceará, Fortaleza, Brasil \\ Emanuela Maria Possidônio de Sousa - Universidade Federal do Ceará, Fortaleza, Brasil
}

\begin{abstract}
Resumo
O presente estudo buscou verificar a adequação dos modelos uni e multifatoriais do AUDIT, bem como verificar sua validade convergente e de critério. Participaram da pesquisa 386 pessoas da população geral com idade média de 27,7 anos $(\mathrm{dp}=10,5)$. Além do AUDIT, os participantes responderam a dois instrumentos correlatos e a perguntas biossociodemográficas. As análises indicaram a adequação do modelo de três fatores, com índices de qualidade de ajuste $\left(\chi^{2} / \mathrm{gl}=63,29, C F I=0,98, A G F I=0,98\right.$ e $\left.R M S E A=0,05\right)$ estatisticamente superiores às estruturas uni e bifatoriais. Finalmente, por meio de correlações $r$ de Pearson e da comparação de médias foram atestadas a validade convergente e de critério do AUDIT, assegurando sua proposta de medir o consumo de álcool e demonstrando sua aplicabilidade na triagem de bebedores-problema.
\end{abstract}

Palavras-chave: AUDIT, Estudo de validação, Análise fatorial confirmatória, Alcoolismo.

Measuring alcohol consumption: confirmatory factor analysis of The Alcohol Use Disorder Identification Test (AUDIT)

\begin{abstract}
This study aims to evaluate the adequacy of uni and multi-factorial models of AUDIT, as well as to verify its convergent and criterion validity. Participants consisted in 386 people from the general population with average age of 27.7 years $(\mathrm{SD}=10.5)$. In addition to AUDIT, the participants responded to two others correlated instruments - CAGE and EAFUA - and to biosocialdemographic questions. The confirmatory factor analysis indicated the adequacy of the model of three factors, with goodness of fit indexes $\left(\chi^{2} / \mathrm{gl}=63,29, \mathrm{CFI}=0,98, \mathrm{AGFI}=0,98\right.$ e RMSEA $\left.=0,05\right)$ statistically higher than the structure of one and two factors. Finally, resorting to Pearson's r correlations and the comparison of means, both convergent and criterion validity of AUDIT were confirmed, attesting its proposal of measuring alcohol consumption and demonstrating its applicability in the screening of problem drinkers.

Keywords: AUDIT, Validation studies, Confirmatory factor analysis, Alcoholism.
\end{abstract}

\section{Midiendo consumo de alcohol: análisis factorial confirmatorio del Alcobol Use Disorder Identification Test (AUDIT)}

\begin{abstract}
Resumen
Este estudio buscó verificar la adecuación de los modelos uni y multi-factoriales del AUDIT y verificar su validez convergente y de criterio. Participaron de la investigación 386 personas de la población general, con un promedio de edad de 27,7 años (DT=10,5). Además del AUDIT, los participantes respondieron a dos instrumentos correlativos y a cuestiones sociodemográficas. Los análisis indicaron la adecuación del modelo de tres factores, con los índices calidad de ajuste $\left(\chi^{2} / \mathrm{gl}=63,29, \mathrm{CFI}=0,98, \mathrm{AGFI}=0,98\right.$ y $\left.\mathrm{RMSEA}=0,05\right)$ estadísticamente superiores a las estructuras uni $\mathrm{y}$ bifactoriales. Finalmente, por medio de correlaciones $r$ de Pearson y de comparación de las medias se comprobó la validez convergente y de criterio del AUDIT, asegurando su propuesta para medir el consumo de alcohol y demostrando su aplicabilidad en la detección de bebedores problema.

Palabras-clave: AUDIT, Estudio de validez, Análisis factorial confirmatorio, Alcoholismo.
\end{abstract}

A triagem para os problemas com o álcool é uma prática relativamente recente, mas tem uma base sólida de apoio, podendo ser realizada em serviços de atenção primária, nas emergências hospitalares, em sistemas judiciários e no meio universitário (National Institute on Alcohol Abuse and Alcoholism - NIAAA, 2005). Em serviços de atenção primária à saúde, por exemplo, recomenda-se a aplicação de instrumentos de triagem para determinar a presença de uso nocivo ou de risco. Nesse contexto, entre os instrumentos de triagem mais citados na literatura, encontram-se o CAGE (Cutdown / Annoyed / Guilty / Eye-opener Questionnaire; Mayfield, McLeod, \& Hall,
1974), o SAAST (Self-Administered Alcoholism Screening Test, Davis Jr., Hurt, Morse, \& O’Brien, 1987), o SMAST (Short Michigan Alcoholism Screening Test, Seltzer, Vinokur, \& van Rooijen, 1975) e o AUDIT (Alcohol Use Disorder Identification Test, Babor, Higgins-Biddle, Saunders, \& Monteiro, 2001).

O Alcohol Use Disorder Identification Test (AUDIT), foco deste estudo, se configura como uma das medidas mais empregadas em todo o mundo para a identificação de grupos de risco e rastreamento do uso inadequado de álcool em amostras clínicas e da população geral (Meneses-Gaya, Zuardi, Loureiro, \& Crippa, 2009; Rist, 
Glöckner-Rist, \& Demmel, 2009; Selin, 2006). Esse instrumento foi originalmente desenvolvido pela Organização Mundial de Saúde no final da década de 1980, com base em um projeto que envolveu seis países (Austrália, Bulgária, Kênia, México, Noruega e Estados Unidos). O estudo contou com uma amostra de 1.888 participantes classificados, a partir de entrevistas prévias, em três grupos: "não bebedores" (36\%), indivíduos abstêmios ou que consumiam bebida alcoólica até três vezes por ano; "bebedores" (48\%), aqueles que consumiam álcool ao menos quatro vezes por ano; e "alcoolistas" (16\%), pessoas com diagnóstico de alcoolismo, já submetidas ou que estavam em busca de tratamento especializado (Saunders, Aasland, Babor, Fuente, \& Grant, 1993).

Os participantes responderam a um conjunto inicial de 150 perguntas que envolviam variáveis de caráter sociobiodemográfico, questões referentes à sua sintomatologia atual, história médica pregressa, consumo de álcool, uso de outras substâncias, reações psicológicas ao álcool, problemas relacionados ao álcool, história familiar de alcoolismo e autopercepção quanto a problemas com álcool. Com base em critérios, como, por exemplo, relevância clínica e cobertura dos domínios teóricos propostos para o AUDIT (frequência do consumo, dependência e consequências negativas do consumo de álcool), juntamente com a capacidade de generalização transcultural do conteúdo, seus proponentes chegaram à versão final composta por dez itens; sete voltados para avaliar o uso nocivo e a dependência de bebidas alcoólicas e três destinados à mensuração da quantidade e da frequência do consumo (Saunders e cols., 1993).

Segundo Rist, Glöckner-Rist, e Demmel (2009), entre os instrumentos utilizados para avaliar o consumo abusivo de álcool, o AUDIT se destaca por sua sensibilidade para detectar a dependência e o consumo nocivo em amostras clínicas e por sua utilidade para identificar potenciais grupos de risco. Ainda que sua utilização tenha sido inicialmente combinada com processos clínicos de triagem, exames laboratoriais e avaliações físicas, a maioria dos estudos atuais o tem empregado como uma medida de triagem (screen) independente (Selin, 2006). Ao longo dos últimos anos, algumas revisões sistemáticas da literatura têm evidenciado a pertinência de sua aplicação com grandes amostras e em contextos culturais distintos (MenesesGaya e cols., 2009; Rist e cols., 2009). Na Suécia, por exemplo, o AUDIT tem sido utilizado em levantamentos nacionais (surveys) acerca do consumo de álcool desde a década de 1990 (Selin, 2006).

De fato, a relevância dessa medida parece comprovada por estudos desenvolvidos em diferentes países do mundo, como: Estados Unidos (Oreskovich e cols., 2012), México (Salcedo, Collazos, Icaza, \& García, 2009), Canadá (Ivis, Adlaf, \& Rehm, 2000), Finlândia (Aalto, Alho, Halme, \& Seppä, 2011), Nigéria (Brisibe, Ordinioha, \& Dienye, 2012), Índia (Carey, Carey, \& Chandra, 2003), Espanha (Gomez, Conde, Santana, \& Jorrin, 2005; Pérula-de Torres e cols., 2005), Suécia (Durbeej e cols., 2010), China (Tsai, Tsai, Chen, \& Liu, 2005), Alemanha (Neumann e cols., 2009), Vietnã (Giang, Spak, Dzung, \& Allebeck, 2005), França (Geneste e cols., 2012) e Austrália (Peng, Wilsnack, Kristjanson, Benson, \& Wilsnack, 2012), entre outros. No entanto, apenas um pequeno número de estudos tem abordado aspectos como validade, estrutura fatorial e confiabilidade dessas versões em idiomas diferentes do inglês, o que compromete sua comparabilidade transcultural (MenesesGaya e cols., 2009; Reinert \& Allen, 2002).

Para o contexto brasileiro, AUDIT foi validado no Rio Grande do Sul por Méndez, Lima, Olinto e Farrel (1999) e, posteriormente, na Bahia por Lima e cols. (2005). No primeiro estudo, não foi efetuada qualquer análise fatorial do instrumento, ao passo que no segundo, desenvolvido na cidade de Salvador, foi possível observar a validade convergente com os critérios da Classificação Internacional de Doenças (CID-10, 2004), consistência interna (alfa de Crombach) e pertinência de uma estrutura fatorial composta por dois fatores (medida do consumo e consequências do consumo de álcool).

Recentemente, Moretti-Pires e Corradi-Webster (2011) buscaram validar o AUDIT para um grupo específico da Amazônia (população ribeirinha). Na ocasião, a versão original foi traduzida para português e, após essa fase, respondida por 361 pessoas. Os resultados indicaram um alfa de Crombach médio de 0,87, considerando três aplicações distintas, e uma correlação de 0,93 entre a primeira e a segunda aplicação (testereteste). Adicionalmente, os autores estabeleceram, por meio da análise de curvas ROC, sete como ponto de corte, obtendo sensibilidade de $76,4 \%$ e especificidade de $75 \%$. No entanto, sem desconsiderar a relevância desses estudos, é preciso destacar que nos últimos dez anos, no Brasil, apenas um estudo (Lima e cols., 2005) ofereceu detalhes acerca da provável estrutura fatorial do AUDIT. Nos demais, o único parâmetro comumente disponibilizado tem sido a consistência interna, o que, por si só, não pode ser avaliado como indicador de validade fatorial do instrumento (Pasquali, 2010), sobretudo, quando não há consenso acerca da estrutura que melhor se ajusta aos dados.

Publicações nessa direção têm indicado modelos compostos por um, dois e até três fatores (Babor, De la Fuente, Saunders, \& Grant, 1992; Carey e cols., 2003; Gmel, Heeb, \& Rehm, 2001; Von der Pahlen e cols., 2008). De acordo com Rist e cols. (2009), os estudos em que se observa a estrutura unifatorial são raros, ao passo 
que os resultados encontrados para modelos que envolvem estruturas com dois e três fatores são mais coesos. Para Meneses-Gaya e cols. (2009), dependendo da amostra, as análises fatoriais efetuadas com matrizes de dados provenientes do AUDIT, em outros países, podem produzir resultados diferentes, justificando-se a necessidade de novos estudos, cujo propósito se volte, fundamentalmente, para a análise e comparação dos diferentes modelos propostos na literatura.

Nesse sentido, o presente estudo teve como principal objetivo comprovar a adequação dos modelos uni e multifatoriais, comparando-os e indicando aquele que melhor se ajusta aos dados, bem como verificar a validade convergente e de critério do AUDIT, considerando uma amostra da população geral. Um detalhamento de suas especificidades pode ser observado no método a seguir.

\section{Método}

\section{Participantes}

Participaram deste estudo 386 pessoas da população geral da cidade de Fortaleza - Ceará, com idades variando entre 18 e 78 anos $(M=27,7$ anos; $D P=10,5)$, a maioria católica $(56,3 \%)$, do sexo feminino (52,2\%), solteira $(74,2 \%)$ e com ensino superior incompleto $(50,9 \%)$. Essa amostra foi de conveniência (não-probabilística), considerando-se as pessoas que concordaram em participar do estudo.

\section{Instrumentos}

Os participantes responderam a um caderno composto pelas seguintes medidas:

Alcohol Use Disorders Identification Test (AUDIT) (Babor e cols., 1992; Saunders e cols., 1993). Como apresentado, esse instrumento é composto por 10 itens, que abrangem três domínios teóricos: (1) consumo de álcool (por exemplo, "Com que frequência você consome alguma bebida alcoólica?"), (2) dependência do consumo de álcool (por exemplo, "Com que frequência, durante o último ano, você achou que não seria capaz de controlar a quantidade de bebida depois de começar?") e (3) consequências adversas do consumo de álcool (por exemplo, "Com que frequência, durante o último ano, você sentiu culpa ou remorso depois de beber?"). Para responder, o participante é convidado a pensar sobre seu consumo de bebidas alcoólicas nos últimos doze meses, indicando sua resposta por meio de diferentes escalas. $\mathrm{O}$ primeiro item envolve a frequência do consumo e é respondido em uma escala de cinco pontos, variando entre 0 (Nunca) e 4 (Quatro ou mais vezes por semana). $\mathrm{O}$ item 2, que mensura a quantidade de bebida alcoólica consumida, inclui cinco opções de respostas que se distribuem entre as opções: não bebo (0); 1 ou 2 "doses"
(1); 3 ou 4 "doses" (2); 5 ou 6 "doses" (3); 7 a 9 "doses" (4); e 10 ou mais "doses" (5). Para os itens 3, 4, 5, 6, 7 e 8, as respostas variam entre os seguintes extremos: Nunca (0) e Todos os dias ou quase todos (4). As questões 9 e 10 apresentam três alternativas de resposta, a saber: Não (0); Sim, mas não no último ano (2); e Sim, durante o último ano (4).

Escala de Atitudes Frente ao Uso de Álcool (EAFUA) (Gouveia, Pimentel, Leite, Albuquerque, \& Costa, 2009). Por meio de escalas e diferencial semântico, o respondente expõe sua avaliação global acerca de estar sob o efeito de álcool. Quatro pares de adjetivos (positivo/negativo, agradável/desagradável, bom/ruim e desejável/indesejável) são situados nos extremos de uma escala composta por nove pontos (bom $+4+3+2+10$ -

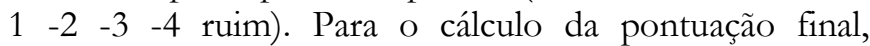
substituem-se os números positivos por 1, 2, 3 e 4 e os negativos por $6,7,8$ e 9 respectivamente, sendo 5 o ponto nulo da escala. Nesse sentido, a maior pontuação indica atitude contrária ao uso de álcool.

Cut down/Annoyed/Guilty/ Eye-opener Questionnaire CAGE (Mayfield e cols., 1974). Amplamente utilizado na detecção de possíveis dependentes de álcool, este instrumento é composto por quatro itens, com alternativas de respostas dicotômicas (sim e não). De acordo com os estudos desenvolvidos no Brasil, duas ou mais respostas afirmativas às seguintes perguntas identificam o potencial bebedor-problema: (1) Alguma vez você sentiu que deveria diminuir ("Cutdown") a quantidade de bebida ou parar de beber? (2) As pessoas o(a) aborrecem ("Annoyed") porque criticam o seu modo de beber? (3) Você se sente culpado(a) ("Guilty)") pela maneira com que costuma beber? (4) Você costuma beber pela manhã para diminuir o nervosismo ou a ressaca ("Eye-opener")? (Amaral \& Malbergiera, 2004; Capriglione, Monteiro, \& Masur, 1985).

A última parte, denominada Caracterização da Amostra, consta de perguntas como sexo, escolaridade, religião, etc., além de questões referentes à experiência com álcool de familiares e pessoas próximas ao respondente. Especificamente, perguntou-se com que frequência essa experiência ocorreu ou tem ocorrido, cuja pergunta é respondida em uma escala de sete pontos, variando de 0 (nunca) a 6 (todos os dias).

\section{Procedimento}

A coleta de dados foi realizada por um grupo de colaboradores treinados para a aplicação padronizada e possíveis esclarecimentos das dúvidas. As pessoas foram abordadas em ruas, praças, shoppings, dentre outros espaços públicos, e convidadas a participar da pesquisa e aquelas que concordavam eram solicitadas a responder o questionário individualmente. $\mathrm{Na}$ ocasião, mediante assinatura de Termo de Consentimento Livre e 
Esclarecido, foi assegurado aos participantes o caráter confidencial de suas respostas e um endereço onde pudessem obter informações sobre os resultados finais do estudo. Além disso, indicou-se que não haveria resposta certa ou errada, que a participação não traria qualquer prejuízo e que os dados seriam tratados no seu conjunto. Em média, 15 minutos foram suficientes para a conclusão do questionário.

\section{Análise dos dados}

Inicialmente, além das estatísticas descritivas, realizadas por meio do SPSS 19, empregou-se o software AMOS 18 para testar diferentes estruturas fatoriais do AUDIT, comparando os índices de ajuste do modelo unifatorial com os de modelos alternativos (multifatoriais). Nessas análises, teve-se em conta a matriz de covariância, adotando o estimador ML (Maximum Likelibood) e observando os seguintes indicadores de ajuste do modelo teórico aos dados empíricos (Byrne, 2001; Tabachnick, Fidell, \& Osterlind, 2001):

(1) Razão $\chi^{2} /$ gl (qui-quadrado/graus de liberdade), sendo recomendáveis valores entre 2 e 3 , porém se admitindo até 5 como indicativo de um ajustamento adequado;

(2) Adjusted Goodness-of-Fit Index (AGFI), cujos valores variam de 0 a 1 , com aqueles próximos a 0,90 indicando um ajustamento satisfatório;

(3) Comparative Fit Index (CFI) é um índice comparativo, adicional, de ajuste ao modelo, valores mais próximos de 1 indicando melhor ajuste, com 0,90 sendo a referência para aceitar o modelo;

(4) Root-Mean-Square Error of Approximation (RMSEA), que se baseia nos residuais, sendo melhor quanto mais próximo este índice for de zero (melhor o ajuste do modelo hipotético aos dados); admitem-se valores de até 0,10 , contudo se tem geralmente em conta o ponto de corte de 0,08 ; e

(5) o Consistent Akaike Information Criterion (CAIC) e o Expected Cross Validation Index (ECVI), os quais são indicadores geralmente empregados para avaliar a adequação de um modelo em relação a outro. Valores baixos do ECVI e CAIC sugerem um modelo mais adequado.

Finalmente, analisou-se a diferença entre os quiquadrados e seus respectivos graus de liberdade $\left[\Delta \chi^{2}(\mathrm{gl})\right]$, procedimento que serve para decidir, entre os modelos, qual o mais parcimonioso e, portanto, mais ajustado
(Byrne, 2001). Ainda com o SPSS, procurou-se conhecer a consistência interna (alfa de Crombach) do AUDIT, bem como evidências de sua validade convergente e de critério (correlação $r_{s}$ de Spearman e teste $t$ de Student) com as medidas correlatas (CAGE e Escala de Atitudes Frente ao Uso de Álcool) ao instrumento.

\section{Resultados}

Para atender aos objetivos do presente estudo, inicialmente, realizou-se uma análise fatorial confirmatória testando a estrutura unifatorial (Modelo 1). De acordo com os resultados da SEM (Structural Equation Modeling), os indicadores de ajuste desse modelo foram pouco aceitáveis: $\chi^{2} \quad(35)=216,86, \quad \mathrm{p}<0,001 ; \quad \chi^{2} / \mathrm{gl}=6,20$, AGFI $=0,82$, CFI $=0,87$, RMSEA $=0,118 \quad(\mathrm{IC} 90 \%=0,103$ 0,133), $\mathrm{ECVI}=0,69$ e $\mathrm{CAIC}=355,34$. Em seguida, com o propósito de identificar um modelo mais adequado, procurou-se, com base na revisão de literatura, testar duas novas estruturas. A primeira (Modelo 2), bifatorial, agrupou o domínio Frequência do Consumo em um único fator, tendo como segundo componente os domínios Dependência e Consequências negativas do Consumo. A segunda (Modelo 3), por outro lado, envolve a versão originalmente proposta pela Organização Mundial de Saúde, em que o consumo de álcool é avaliado a partir dos três domínios supracitados. Os resultados dessas análises são sumarizados na Tabela 1 .

Quando comparados, o Modelo 2 foi mais apropriado $\left(\chi^{2} / \mathrm{gl}=101,80, \quad \mathrm{AGFI}=0,92, \quad \mathrm{CFI}=0,95 \quad \mathrm{e}\right.$ RMSEA $=0,07)$ que o Modelo 1. Entretanto, considerando os múltiplos indicadores de qualidade de ajuste, percebe-se que o Modelo 3 reúne provas de melhor adequação $\quad\left(\chi^{2} / \mathrm{gl}=63,29, \quad\right.$ AGFI $=0,98 \quad \mathrm{e}$ RMSEA $=0,05)$ ante do Modelo 2 e, consequentemente, do Modelo 1. Além disso, os valores do CAIC e do ECVI no Modelo 3 são inferiores aos observados nos demais modelos, sendo significativa a diferença de qui-quadrado dos mesmos, principalmente quando se observam os Modelos 3 e $2\left(\Delta \chi^{2}(2)=38,51, p<0,001\right)$. Em virtude dos resultados, assume-se o Modelo 3 como o mais adequado. Nesse caso, excetuando o item 6 , todos os $\lambda$ foram superiores a 0,30 , sendo estatisticamente diferentes de zero $(\lambda \neq 0 ; \quad z>1,96, \quad p<0,05)$. Os três fatores apresentaram correlação entre si $(\Phi)$ de $0,53,0,68$ e 0,70 (valor Phi padronizado). Um resumo desse modelo pode ser observado na Figura 1. 
Tabela 1. Comparação de diferentes modelos fatoriais do AUDIT

\begin{tabular}{cccccccc}
\hline Modelo & $\chi^{2}(\mathrm{gl})$ & AGFI & CFI & RSMEA $($ CI90\%) & ECVI & CAIC & $\Delta \chi^{2}(\mathrm{gl})$ \\
\hline 1 & $216,86(35)$ & 0,82 & 0,87 & $0,12(0,10-0,13)$ & 0,69 & 355,34 & ---- \\
2 & $101,80(34)$ & 0,92 & 0,95 & $0,07(0,06-0,09)$ & 0,39 & 247,21 & $115,06(1)^{*}$ \\
3 & $63,29(32)$ & 0,95 & 0,98 & $0,05(0,03-0,07)$ & 0,29 & 222,54 & $38,51(2)^{*}$ \\
\hline
\end{tabular}

Nota: $\chi^{2}=$ qui-quadrado, $\mathrm{gl}=$ graus de liberdade, $\mathrm{AGFI}=$ Adjusted goodness-of-fit index, $\mathrm{CFI}=$ comparative fit index, RMSEA=root-mean-square error of approximation, CI90\%=intervalo de confiança de $90 \%$, ECVI=expected cross-validation index, CAIC $=$ consistente Akaikein formation criterion, e $\Delta \chi^{2}=$ diferença entre os valores do qui-quadrado. ${ }^{*} p<0,001$.

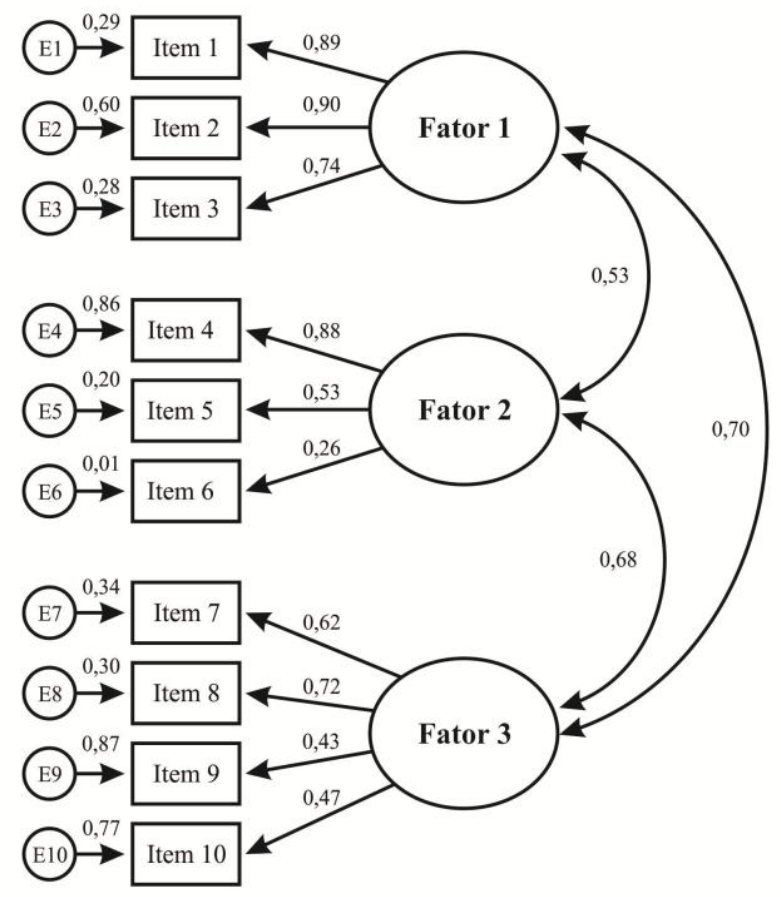

Figura 1. Estrutura fatorial do Alcohol Use Disorder Identification Test (AUDIT)

Adicionalmente, procurou-se calcular a fidedignidade do AUDIT, considerando o conjunto de itens. Os resultados dessas análises indicaram consistência interna (alfa de Crombach) de 0,82, com homogeneidade média de 0,52 , variando de 0,25 (item 6: Com que frequência depois de ter bebido muito, você precisou beber pela manhã para se sentir melhor?) a 0,76 (item 3: Com que frequência você toma "seis ou mais doses" em uma ocasião?). Entretanto, como os resultados indicaram a coerência do Modelo 3, calcularam-se os alfas de Crombach para cada um dos fatores desse modelo.

Para o primeiro fator (frequência de consumo), observaram-se alfa de 0,89 e índice de homogeneidade médio de 0,83 (correlação item-total média corrigida), variando este último entre 0,81 (item 1: Com que frequência você consome alguma bebida álcoolica?) e 0,85 (item 2: Nas ocasiões em que bebe, quantas doses, copos ou garrafas você costuma tomar?). O Segundo Psico-USF, Bragança Paulista, v. 18, n. 1, p. 121-130, jan./abril 2013 fator (dependência) apresentou consistência interna e homogeneidade média de 0,50 e 0,39 , respectivamente. Para esse fator, o menor índice de homogeneidade foi de 0,20 (item 6: Com que frequência depois de ter bebido muito, você precisou beber pela manhã para se sentir melhor?) e o maior 0,51 (item 4: Com que frequência você achou que não seria capaz de controlar a quantidade de bebida depois de começar?). O último componente (consequências negativas do consumo) apresentou um alfa de 0,62 e homogeneidade média de 0,42 , sendo o valor mais baixo $(0,36)$ correspondente ao item 10 (Alguma vez na vida algum parente, amigo, médico ou outro profissional da saúde já se preocupou com você por causa de bebida ou lhe disse para parar de beber?) e mais alto $(0,48)$ ao item 8 (Com que frequência você não conseguiu se lembrar do que aconteceu na noite anterior por causa da bebida?). Adicionalmente, em função da natureza das análises realizadas no presente estudo, decidiu-se observar, 
ainda, a confiabilidade composta para cada um dos fatores do AUDIT. Os três fatores (consumo de álcool, dependência do consumo de álcool e consequências adversas do consumo de álcool) apresentaram confiabilidade composta de 0,88, 0,61 e 0,65, respectivamente.

Finalmente, a fim de avaliar a validade convergente do AUDIT, procurou-se correlacionar sua pontuação total e as dos seus respectivos fatores com aquelas obtidas por meio da Escala de Atitudes Frente ao Uso de Álcool (Gouveia e cols., 2009) e do
Cutdown/ Annoyed/ Guilty/ Eye-opener Questionnaire (CAGE; Mayfield e cols., 1974). Tal análise se justifica, fundamentalmente, pela relação existente entre atitudes e comportamento e, principalmente, atitudes ante o consumo de álcool e ingestão de bebidas alcoólicas, bem como entre as pontuações do CAGE AUDIT (Ajzen, 2001; Hays, Merz, \& Nicholas, 1995; Holland, Verplanken, \& Van Knippenberg, 2002; Simons \& Carey, 2000; Simons \& Gaher, 2004). Os resultados dessas análises são apresentados na Tabela 2.

Tabela 2. Correlação de Spearman entre AUDIT, CAGE e Atitudes Frente ao Uso de Álcool

\begin{tabular}{|c|c|c|c|c|c|}
\hline \multirow{2}{*}{\multicolumn{6}{|c|}{$\frac{\text { Variáveis }}{1 . \text { CAGE }}$}} \\
\hline & & & & & \\
\hline 2. Atitudes Frente ao Uso de Álcool & $-0,19^{*}$ & & & & \\
\hline 3. AUDIT total & $0,55^{*}$ & $-0,52^{*}$ & & & \\
\hline 4. Frequência de consumo & $0,46^{*}$ & $-0,58^{*}$ & $0,93^{*}$ & & \\
\hline 5. Dependência & $0,44^{*}$ & $-0,24^{*}$ & $0,66^{*}$ & $0,52^{*}$ & \\
\hline 6. Consequência negativas do consumo & $0,53^{*}$ & $-0,34^{*}$ & $0,83^{*}$ & $0,66^{*}$ & $0,51^{*}$ \\
\hline
\end{tabular}

Nota: ${ }^{*} p \leq 0,01 ; \mathrm{CAGE}=$ Cut down / Annoyed / Guilty / Eye-opener Questionnaire; AUDIT = Alcohol Use Disorder Identification Test

Como proposto na literatura (Hays e cols., 1995), o consumo de álcool, avaliado pelo AUDIT, está correlacionado diretamente às pontuações dos participantes no CAGE $\left(r_{s}=0,55 ; p \leq 0,01\right)$. As atitudes ante o consumo de álcool se correlacionam negativa e significativamente, tanto com a pontuação total do AUDIT $(r=-0,52 ; p \leq 0,01)$ como com os fatores teóricos propostos $(r=-0,58, \quad-0,24$ e $-0,34$ respectivamente; $p \leq 0,01)$. Além disso, quando se avaliam as pontuações médias dos participantes no AUDIT, comparando potenciais bebedores-problema $(M=1,32)$, selecionados por meio do CAGE, e os respondentes que não se enquadram nessa categoria de consumidores de álcool $(M=0,98)$, verifica-se que estes se diferenciam significativamente daqueles $(t=3,14$; $p \leq 0,01)$, o que ratifica a validade de critério do AUDIT. Tais resultados são discutidos mais detalhadamente a seguir.

\section{Discussão}

Como apresentado, este estudo teve como objetivo comprovar a adequação dos modelos uni e multifatoriais do AUDIT, verificando sua convergência com construtos correlatos e sua validade de critério. Em termos gerais, observando os resultados, a análise fatorial indicou o modelo de três fatores como o de melhor adequação, o que corrobora a proposta dos três domínios sugerida pela OMS (Babor e cols., 2001). Acerca dessa semelhança, é preciso destacar que, originalmente, o estabelecimento desses domínios teve por base apenas a validade aparente, que, em si, não garante a validade fatorial do instrumento (Pasquali, 2010). Desse modo, a comprovação apresentada, por meio dos índices de ajuste, forneceu indícios empíricos da adequação deste modelo. Segundo Lima e cols. (2005), em amostras procedentes de populações com alta prevalência de dependência, os resultados podem indicar a pertinência do modelo unifatorial, entretanto, aquelas em que se observa baixa prevalência de transtornos relacionados com o consumo de álcool, remetem à estrutura composta por dois ou três fatores. Esse aspecto pôde ser comprovado no presente estudo, uma vez que os dados utilizados são provenientes de pessoas da população geral, moradores de diferentes regiões de uma capital brasileira.

Em relação à consistência interna (alfa de Crombach), ainda que se reconheça que esse parâmetro nos fatores dependência $(a=0,50)$ e consequências negativas $(a=0,62)$ não esteja dentro dos valores sugeridos como desejáveis $(a \geq 0,70$; Nunnally \& Bernstein, 1995), é preciso considerar alguns aspectos importantes que podem influenciar esse índice, como, por exemplo, o tamanho da amostra e o número de itens em cada fator. Além disso, deve-se ponderar que os itens que abrangem os fatores dependência e consequências negativas envolvem aspectos referentes aos sintomas de dependência e às consequências adversas do consumo nocivo de álcool, características que, na amostra utilizada, não apresentam muita variabilidade. Não obstante, cabe assinalar que, para instrumentos de screen e utilizados em estudos 
exploratórios, alfas próximos a 0,60 podem ser considerados aceitáveis (Clark \& Watson, 1995). Esse aspecto pode ser reforçado por meio da confiabilidade composta, índice que não sofre influência do número de itens e que, segundo a literatura, admite como razoáveis valores a partir de 0,60 (Fornell \& Larcker, 1981; Hair, Black, Babin, Anderson, \& Tatham, 2009).

Acerca da baixa consistência interna do fator dependência, análises adicionais revelaram que, caso o item 6 fosse excluído, o valor do alfa de Crombach iria de 0,50 para 0,58. Entretanto, atendendo aos requisitos da SEM (Structural Equation Modeling) de considerar ao menos três indicadores por fator, a exclusão desse item poderia comprometer as análises (Gefen, Straub, \& Boudreau, 2000; Hair e cols., 2009). Deve-se observar que o item 6 , apesar de apresentar $\lambda$ inferior a 0,30 , foi estatisticamente diferente de zero $(\lambda \neq 0 ; \quad z>1,96$, $p<0,05)$. Ao mesmo tempo, é preciso perceber que seu conteúdo abrange um dos principais aspectos que caracterizam o sintoma de dependência. Nesse caso, uma vez que o AUDIT é uma medida de screen, a exclusão do item 6 prejudicaria a dimensionalidade do fator dependência, pois o deixar com apenas dois itens não seria suficiente para garantir a definição do construto. Nesse sentido, torna-se plausível sugerir, por um lado, a permanência do item, assumindo o comprometimento da definição operacional da variável medida e, por outro, o que é mais indicado, a modificação do mesmo em estudos futuros, deixandoo mais próximo da realidade de participantes oriundos da população não-clínica.

Por fim, os resultados fazem referência, ainda, à validade convergente e a de critério. Para se avaliar tais validades, as pontuações médias no AUDIT foram correlacionadas às da Escala de Atitudes Frente ao Uso de Álcool e comparadas em função de grupos critério, como definidos pelo CAGE (bebedor-problema vs. social). Como visto, os resultados asseguraram a proposta do AUDIT em medir o consumo de álcool e demonstraram sua aplicabilidade na triagem de bebedores-problema.

Em síntese, confia-se que o objetivo do presente estudo, a saber, observar a adequação dos modelos uni e multifatoriais do AUDIT, comparando-os e identificando aquele com melhor ajuste aos dados, e verificar sua validade convergente e de critério, tenha sido alcançado satisfatoriamente. Embora pareçam evidentes as contribuições desta pesquisa, é possível apontar algumas limitações potenciais. No primeiro momento, deve-se reconhecer que tais resultados abrangem unicamente informações resultantes de uma amostra de conveniência, não representativa da população, o que limita a generalização dos resultados.
Sobre esse tema, Laros e Pasquali (2005) afirmam que, quando uma escala é designada para uso clínico, a exemplo do AUDIT, torna-se de grande importância a utilização de amostras clínicas para sua confirmação fatorial.

Diante do exposto, sugerem-se novos estudos, nos quais seja considerada a possibilidade de ampliação da amostra, que garanta a representatividade de toda a extensão da população-alvo, envolvendo, além da população geral, grupos mais específicos, como é o caso de pacientes diagnosticados como bebedoresproblema. Tal procedimento poderá garantir maior heterogeneidade da amostra, tornando mais evidente a adequação da estrutura fatorial do AUDIT. Essas ações poderão dirimir possíveis dúvidas acerca das consistências internas de dois dos seus componentes, como é o caso do fator dependência.

Finalmente, ainda que o presente estudo não tenha cunho eminentemente prático, não se pode deixar de considerar que os dados aqui disponibilizados podem ser úteis em diversos campos, a exemplo dos estudos epidemiológicos, nos quais ferramentas como entrevistas nem sempre são aplicáveis pelo tamanho da amostra. Dada a sua brevidade, o AUDIT surge como uma boa alternativa na detecção de bebedores problema e como ferramenta auxiliar na implementação de programas de prevenção e/ou intervenção de uso abusivo de álcool. Ademais, esperase que os resultados aqui apresentados contribuam para a ampliação de informações acerca das propriedades psicométricas do AUDIT, expandindo a confiança na sua utilização e permitindo a replicabilidade das pesquisas, tanto no âmbito nacional como no internacional.

\section{Referências}

Aalto, M., Alho, H., Halme, J. T., \& Seppä, K. (2011). The alcohol use disorders identification test (AUDIT) and its derivatives in screening for heavy drinking among the elderly. International Journal of Geriatric Psychiatry, 26(9), 881-885.

Ajzen, I. (2001). Nature and operation of attitudes. Annual Review of Psychology, 52(1), 27-58.

Amaral, R. A., \& Malbergiera, A. (2004). Avaliação de instrumento de detecção de problemas relacionados ao uso do álcool (CAGE) entre trabalhadores da prefeitura do campus da Universidade de São Paulo (USP)-campus capital; Evaluation of a screening test for alcohol-related problems (CAGE) among employees of the Campus of the University of São Paulo. Rev. Bras. Psiquiatr, 26(3), 156-163. 
Babor, T. F., De la Fuente, J., Saunders, J., \& Grant, M. (1992). Audit. The Alcohol Use Disorders Identification Test. Guidelines for use in primary bealth care. Geneva, Switzerland: World Health Organization.

Babor, T. F., Higgins-Biddle, J. C., Saunders, J. B., \& Monteiro, M. G. (2001). The alcohol use disorders identification test. World Health Organization (Geneva).

Brisibe, S., Ordinioha, B., \& Dienye, P. O. (2012). Intersection between alcohol abuse and intimate partner's violence in a rural ijaw community in Bayelsa State, South-South Nigeria. Journal of Interpersonal Violence, 27(3), 513-522.

Byrne, B. M. (2001). Structural equation modeling with AMOS: basic concepts, applications, and programming.

Capriglione, M. J., Monteiro, M. G., \& Masur, J. (1985). Aplicaçäo do questionario CAGE para detecçäo da síndrome de dependência do álcool em 700 adultos na cidade de Säo Paulo; application of CAGE questionnaire for detection of alcohol dependence syndrome in 700 adults in the city of Säo Paulo. Rev. Assoc. Bras. Psiquiatr, 7(25), 50-53.

Carey, K. B., Carey, M. P., \& Chandra, P. S. (2003). Psychometric evaluation of the alcohol use disorders identification test and short drug abuse screening test with psychiatric patients in India. The Journal of Clinical Psychiatry, 64(7), 767.

Clark, L. A., \& Watson, D. (1995). Constructing validity: basic issues in objective scale development. Psychological Assessment, 7(3), 309.

Davis Jr, L. J., Hurt, R. D., Morse, R. M., \& O'Brien, P. C. (1987). Discriminant Analysis of the Self-Administered Alcoholism Screening Test. Alcoholism: Clinical and Experimental Research, 11(3), 269-273.

Durbeej, N., Berman, A. H., Gumpert, C. H., Palmstierna, T., Kristiansson, M., \& Alm, C. (2010). Validation of the Alcohol Use Disorders Identification Test and the Drug Use Disorders Identification Test in a Swedish sample of suspected offenders with signs of mental health problems: Results from the Mental Disorder, Substance abuse and Crime study. Journal of Substance Abuse Treatment, 39(4), 364-377.

Fornell, C., \& Larcker, D. F. (1981). Evaluating structural equation models with unobservable variables and measurement error. Journal of marketing research, 39-50.

Gefen, D., Straub, D. W., \& Boudreau, M. C. (2000). Structural equation modeling and regression: guidelines for research practice.
Geneste, J., Pereira, B., Arnaud, B., Christol, N., Liotier, J., Blanc, O., . . . Llorca, P. (2012). CAGE, RAPS4, RAPS4-QF and AUDIT Screening Tests for Men and Women Admitted for Acute Alcohol Intoxication to an Emergency Department: Are Standard Thresholds Appropriate? Alcohol and Alcobolism, 47(3), 273-281.

Giang, K. B., Spak, F., Dzung, T. V., \& Allebeck, P. (2005). The use of AUDIT to assess level of alcohol problems in rural Vietnam. Alcohol and Alcoholism, 40(6), 578-583.

Gmel, G., Heeb, J. L., \& Rehm, J. (2001). Is frequency of drinking an indicator of problem drinking? A psychometric analysis of a modified version of the alcohol use disorders identification test in Switzerland. Drug and Alcohol Dependence, 64(2), 151-163.

Gomez, A., Conde, A., Santana, J., \& Jorrin, A. (2005). Diagnostic usefulness of brief versions of Alcohol Use Disorders Identification Test (AUDIT) for detecting hazardous drinkers in primary care settings. J Stud Alcohol, 66(2), 305-308.

Gouveia, V. V., Pimentel, C. E., Leite, P. R. L., Albuquerque, J. R., \& Costa, T. A. B. (2009). Escala de atitudes frente ao uso de álcool: descrevendo seus parâmetros psicométricos. Psicologia: Ciência e Profissão, 29(4), 672-685.

Hair, J. F., Black, W. C., Babin, B. J., Anderson, R. E., \& Tatham, R. L. (2009). Análise multivariada de dados. Porto Alegre: Bookman.

Hays, R. D., Merz, J. F., \& Nicholas, R. (1995). Response burden, reliability, and validity of the CAGE, Short MAST, and AUDIT alcohol screening measures. Behavior Research Methods, 27(2), 277-280.

Holland, R. W., Verplanken, B., \& Van Knippenberg, A. (2002). On the nature of attitude-behavior relations: the strong guide, the weak follow. European Journal of Social Psychology, 32(6), 869-876.

Ivis, F. J., Adlaf, E. M., \& Rehm, J. (2000). Incorporating the AUDIT into a general population telephone survey: a methodological experiment. Drug and Alcohol Dependence, 60(1), 97104.

Laros, J., \& Pasquali, L. (2005). O uso da análise fatorial: algumas diretrizes para pesquisadores. Em L. Pasquali (Org.). Análise fatorial para pesquisadores (pp. 147-170). Petrópolis: Vozes.

Lima, C. T., Freire, A. C. C., Silva, A. P. B., Teixeira, R. M., Farrell, M., \& Prince, M. (2005). Concurrent and construct validity of the AUDIT in an urban Brazilian sample. Alcohol and Alcoholism, 40(6), 584589. 
Mayfield, D., McLeod, G., \& Hall, P. (1974). The CAGE questionnaire: validation of a new alcoholism screening instrument. The American Journal of Psychiatry, 131(10), 1121-1123.

Méndez, E. B., Lima, M., Olinto, M., \& Farrel, M. (1999). Uma versão brasileira do AUDIT-Alcohol Use Disorders Identification Test. Pelotas: Universidade Federal de Pelotas.

Meneses-Gaya, C., Zuardi, A. W., Loureiro, S. R., \& Crippa, J. A. S. (2009). Alcohol Use Disorders Identification Test (AUDIT): an updated systematic review of psychometric properties. Psychology \&amp; Neuroscience, 2(1), 83-97.

Moretti-Pires, R. O., \& Corradi-Webster, C. M. (2011). Adaptação e validação do Alcohol Use Disorder Identification Test (AUDIT) para população ribeirinha do interior da Amazônia, Brasil Adaptation and validation of the Alcohol Use Disorders Identification Test (AUDIT) for. Cad. Saúde Pública, 27(3), 497-509.

National Institute on Alcohol Abuse and Alcoholism. (2005). Screening for alcohol use and alcoholrelated problems. Retrieved 15 de maio de 2012, from

http://pubs.niaaa.nih.gov/publications/aa65/AA 65.htm

Neumann, T., Gentilello, L. M., Neuner, B., Weiß-Gerlach, E., Schürmann, H., Schröder, T., Spies, C. D. (2009). Screening trauma patients with the Alcohol Use Disorders Identification Test and biomarkers of alcohol use. Alcoholism: Clinical and Experimental Research, 33(6), 970-976.

Nunnally, J. C., \& Bernstein, I. J. (1995). Teoría psicométrica: México: McGraw Hill.

Oreskovich, M. R., Kaups, K. L., Balch, C. M., Hanks, J. B., Satele, D., Sloan, J., Shanafelt, T. D. (2012). Prevalence of alcohol use disorders among American surgeons. Archives of Surgery, 147(2), 168.

Pasquali, L. (2010). Instrumentação psicológica: Fundamentos e prática. Porto Alegre: Artmed. Textos recomendados: Texto, 1, 11-21.

Peng, C. Z., Wilsnack, R. W., Kristjanson, A. F., Benson, P., \& Wilsnack, S. C. (2012). Gender differences in the factor structure of the Alcohol Use Disorders Identification Test in multinational general population surveys. Drug Alcohol Dependence, 1(124), 1-2.

Pérula-de Torres, L., Fernández-García, J., Arias-Vega, R., Muriel-Palomino, M., Márquez-Rebollo, E., \& Ruiz-Moral, R. (2005). Validity of AUDIT test for detection of disorders related with alcohol consumption in women. Medicina Clínica, 125(19), 727.
Reinert, D. F., \& Allen, J. P. (2002). The alcohol use disorders identification test (AUDIT): a review of recent research. Alcoholism: Clinical and Experimental Research, 26(2), 272-279.

Rist, F., Glöckner-Rist, A., \& Demmel, R. (2009). The Alcohol Use Disorders Identification Test revisited: establishing its structure using nonlinear factor analysis and identifying subgroups of respondents using latent class factor analysis. Drug and Alcohol Dependence, 100(1-2), 71-82.

Salcedo, V. V., Collazos, M. V., Icaza, M. E., \& García, F. J. (2009). Validation of the alcohol use disorders identification test (AUDIT) in Mexican patients with schizophrenia. Revista Panamericana de Salud Pública, 26(4), 283-289.

Saúde, O. P. d., \& Organization, P. A. H. (2004). Classificação estatística internacional de doenças e problemas relacionados à saúde. São Paulo: Edusp.

Saunders, J. B., Aasland, O. G., Babor, T. F., Fuente, J. R., \& Grant, M. (1993). Development of the alcohol use disorders identification test (AUDIT): WHO collaborative project on early detection of persons with harmful alcohol consumption-II. Addiction, 88(6), 791-804.

Selin, K. H. (2006). Alcohol Use Disorder Identification Test (AUDIT): what does it screen? Performance of the AUDIT against four different criteria in a Swedish population sample. Substance Use \& Misuse, 41(14), 1881-1899.

Seltzer, M., Vinokur, A., \& Van Rooijen, L. (1975). A self-administered Short Michigan Alcoholism Screening Test (SMAST). J Stud Alcohol, 36(117), 26.

Simons, J. S., \& Gaher, R. M. (2004). Attitudes toward alcohol and drug-free experience among college students: Relationships with alcohol consumption and problems. The American Journal of Drug and Alcobol Abuse, 30(2), 461-471.

Simons, J., \& Carey, K. B. (2000). Attitudes toward marijuana use and drug-free experience* $1::$ relationships with behavior. Addictive Behaviors, 25(3), 323-331.

Tabachnick, B. G., Fidell, L. S., \& Osterlind, S. J. (2001). Using multivariate statistics. New York: Allyn \& Bacon.

Tsai, M. C., Tsai, Y. F., Chen, C. Y., \& Liu, C. Y. (2005). Alcohol Use Disorders Identification Test (AUDIT): establishment of cut-off scores in a hospitalized chinese population. Alcoholism: Clinical and Experimental Research, 29(1), 53-57.

Von der Pahlen, B., Santtila, P., Witting, K., Varjonen, M., Jern, P., Johansson, A., \& Sandnabba, N. K. (2008). Factor structure of the Alcohol Use 
Disorders Identification Test (AUDIT) for men and women in different age groups. Journal of Studies on Alcohol and Drugs, 69(4), 616.

Recebido em: 28/06/2012 Reformulado em: 29/01/2013

Aprovado em: 26/02/2013

Sobre os autores:

Walberto Silva dos Santos é doutor em Psicologia Social pela Universidade Federal da Paraíba, professor adjunto II na Universidade Federal do Ceará.

Darlene Pinho Fernandes é mestranda do Programa de Pós-Graduação (Mestrado) em Psicologia da Universidade Federal do Ceará.

Alex Sandro de Moura Grangeiro é mestrando do Programa de Pós-Graduação (Mestrado) em Psicologia da Universidade Federal do Ceará.

Guilherme Sobreira Lopes é bolsista de Iniciação à Docência da Universidade Federal do Ceará.

Emanuela Maria Possidônio de Sousa é bolsista de Iniciação Científica da Universidade Federal do Ceará.

Contato com os autores:

Universidade Federal do Ceará

$\mathrm{CH}$ - Departamento de Psicologia

Av. da Universidade, 2762 - CEP 60020-180 - Fortaleza/CE, Brasil

E-mail: walbertosantos@ufc.br 